1

2

3

\section{Abstract}

- The apparent moments of inertia of Callisto and Titan inferred from gravity 7 data suggest incomplete differentiation of their interior, commonly attributed

8 to slow and cold accretion. To understand whether such large icy moons can 9 really avoid global melting and subsequent differentiation during their accre-

\author{
J. Monteux ${ }^{\mathrm{a}}$, G. Tobie ${ }^{\mathrm{a}}$, G. Choblet ${ }^{\mathrm{a}}$, and M. Le Feuvre ${ }^{\mathrm{b}}$ \\ ${ }^{a}$ Laboratoire de Planétologie et de Géodynamique de Nantes \\ ${ }^{b}$ Laboratoire Auscultation et Imagerie, IFSTTAR, Nantes
} tion, we have developed a 3D numerical model that characterizes the thermal evolution of a satellite growing by multi-impacts, simulating the satellite growth and thermal evolution for a body radius ranging from 100 to 2000 kilometers. The effects of individual impacts (energy deposition, excavation) are simulated and integrated for impactor sizes ranging from a few kilometers to one hundred kilometers, while for smaller impactors, a simplified approach with successive thin uniform layers spreading all over the satellite is considered. Our simulations show that the accretion rate plays only a minor role and that extending the duration of accretion does not significantly limit the increase of the internal temperature. The mass fraction brought by large impactors plays a more crucial role. Our results indicate that a satellite exceeding $2000 \mathrm{~km}$ in radius may accrete without experiencing significant melting only if its accretion is dominated by small impactors ( $<$ a few kilometers) and that the conversion of impact energy into heat is unrealistically inefficient $(<10-15 \%)$. Based on our simulations, if more than $10 \%$ of satellite mass was brought by satellitesimals larger than $1 \mathrm{~km}$, global melting for large bodies like Titan or Callisto cannot be avoided.

\title{
Can Large Icy Moons Accrete Undifferentiated?
}


${ }_{27}$ Key words: Thermal histories; Accretion; Satellites, formation; Impact processes 
42

43

44

45

\section{Introduction}

Differences in composition and internal structure exist between the major icy satellites of Jupiter and Saturn, suggesting distinct accretion and differentiation histories (e.g., Kirk and Stevenson, 1987; Mueller and McKinnon, 1988; Mosqueira and Estrada, 2003a; Barr and Canup, 2008). The high moment of inertia factor inferred from Galileo gravity measurements $\left(\mathrm{C} / \mathrm{MR}^{2}=0.346\right)(A n$ derson et al., 2001) suggests that ice-rock separation may be incomplete in the interior of Jupiter's moon Callisto. By contrast, Ganymede has a much smaller moment of inertia $\left(\mathrm{C} / \mathrm{MR}^{2}=0.31\right)$ (Anderson et al., 2001) and shows signs of past endogenic activity (Pappalardo et al., 2004). A full separation of ice and rock is suggested for Ganymede together with the formation of a metallic core, which is at the origin of a relatively intense intrinsic magnetic field (Kivelson et al., 1998).

With similar size and mass, Saturn's moon Titan may be an intermediate case between Callisto and Ganymede. Its moment of inertia factor, C/MR ${ }^{2}$ estimated to $\sim 0.33-0.34$ from Cassini gravity measurements (Iess et al., 2010, 2012)) suggests that Titan's interior is more differentiated than Callisto but probably much less than Ganymede. Like Callisto, Titan might still possess a layer of ice-rock mixture between a rocky core and a outer ice-rich mantle, unless the rocky core is mostly composed of highly hydrated minerals (Sohl et al., 2010; Castillo-Rogez and Lunine, 2010). The fact that the interior of Callisto and possibly Titan may still contain a layer of ice-rock mixture suggests that the satellite may have avoided significant melting during accretion and subsequent evolution.

The accretion of giant planet's moons is intimately linked with the evo- 
lution of the circumplanetary disk that formed during the transition stage of the planet's accretion, when the planet became massive enough to contract and accrete gas and dust from the circumsolar disk (e.g., Estrada et al., 2009). The timescale of the satellite accretion is therefore mostly controlled by the disk structure, the mass inflow rate, and the lifetime of the circumplanetary disk. Two main categories of circumplanetary disk models have been proposed: the solids-enhanced minimum mass (SEMM) model (Mosqueira and Estrada, 2003a,b; Estrada et al., 2009) and the gas-starved disk model (Canup and Ward, 2002, 2006; Ward and Canup, 2010). In the gas-starved disk model, the disk is assumed to be continuously supplied by ongoing inflow of gas and dust particles from the surrounding proto-planetary disk while in the SEMM model, solid components of the disk are supplied by ablation and capture of planetesimal fragments passing through the disk. These two approaches result in different characteristic impactor sizes, ranging typically from a few meters to a few kilometres in the gas-starved approach (Barr and Canup, 2008), while a significant fraction of impactors with radii above $1 \mathrm{~km}$ size and up to $100-200 \mathrm{~km}$ is envisioned in the SEMM model (Estrada and Mosqueira, 2011). The impactor size is crucial to determine whether the impact energy is buried deep beneath the surface or efficiently released to the space. Hence these two formation models can potentially lead to different early thermal evolutions of growing icy moons.

Previous studies showed that it was possible to avoid melting if the accumulation of accretion energy was inefficient, i.e. if the energy was radiated away at a rate comparable to the accretion rate (e.g., Schubert et al., 1981; Squyres et al., 1988; Kossacki and Leliwa-Kopystyński, 1993; Coradini et al., 1995; Grasset and Sotin, 1996; Barr and Canup, 2008; Barr et al., 2010). Based on these models, the accretion timescales $t_{a c c}$ should be longer than 1 Myr to avoid significant 
83

84 short as $10^{3-4}$ yr may be possible for Ganymede. However, these timescales are

85

melting and hence differentiation of Callisto while an accretion timescale as dependent on the way heat deposition and cooling are treated. These studies used an one-dimensional approach initially developed for the accretion of terrestrial planets (Safronov, 1978; Kaula, 1979). In this approach, the evolution is parameterized by deposition of successive material layers. The thermal effect of an impact is not considered individually, but is averaged over the entire surface and integrated. This approach is valid as long as the impactors remain small $(\leq 1 \mathrm{~km})$ and are randomly distributed at the surface. This might be the case during the very early stage of the accretion process, but impactors larger than $1 \mathrm{~km}$ probably became more and more abundant at the end of the accretion stage (e.g., Estrada et al., 2009). Impactors larger than $100 \mathrm{~km}$ might also be expected (e.g., Sekine and Genda, 2012; Dwyer et al., 2013). For such large impacts, a detailed description of each impact including energy deposition and transfer is required.

For this purpose, we have developed a three-dimensional model that characterizes the thermal evolution of a satellite growing by multi-impacts. The satellite growth and thermal evolution are simulated for a radius ranging from 100 kilometers to $2000 \mathrm{~km}$ from different populations of undifferentiated icy impactors, by assuming different accretion rates and conversion rates of impact energy into heat. The effects of individual impacts are simulated and integrated for impactor sizes ranging from a few kilometers to one hundred kilometers. For each impact event, we consider the thermal effects due to the dissipation of the impactor's kinetic energy as heat as well as the topographical effect associated to excavation process. For impactor sizes smaller than a few kilometers, we do not treat the impact individually because the number of impacts to simulate will 
be extremely time consuming. The small and numerous impactors are modeled by successive thin uniform layers spreading all over the moon. As the icy moon grows, gravitational forces increase and impacts become more and more violent.

Due to this, as well as the accumulation of warmed icy material, melting events may occur once the icy moon reaches a critical size. As the main objective of our work is to determine the maximum radius reached by a growing satellite before significant melting occurs $(>5 \%)$, we make some simple assumptions corresponding to the least efficient scenario for initiating ice melting. The impacts are assumed to occur with the smallest possible velocity corresponding to the escape velocity determined by the mass of the growing satellite. Hence, the accretion efficiency is assumed to be $100 \%$ and all impacted mass remains on the growing satellite (Asphaug, 2010). With these assumptions, we minimize the energy accumulated in the satellite during the growth, and therefore we provide an upper limit for the radius that the satellite can reach without experiencing significant melting. In sections 2 and 3, we present the details of our model. We first describe the process associated to a single impact event and then we present our multi-impact approach. The results of our simulations for different accretion parameters are provided in Section 4. Finally, in section 5, we briefly discuss the implications of our results for the post-accretionnal structure of large icy moons and the subsequent differentiation processes.

\section{Single impact model}

Following an impact and the formation of a crater, a significant amount of heat is buried deep below the impact site. In the following section we describe the scaling laws used to model the thermal and topographical consequences of a large single impact on a growing icy moon. 


\subsection{Impact heating}

During an impact event, the initial kinetic energy of the impactor is converted into internal energy produced by shock heating of the satellite and of the impactor, internal energy produced by plastic work, and kinetic energy of ejected material (e.g O'Keefe and Ahrens, 1977; Squyres et al., 1988). O'Keefe and Ahrens (1977) estimated that the fraction, $\gamma_{l i}$, of the impactor kinetic energy going into shock heating of the satellite ranged from 0.2 for low-velocity impacts to about 0.6 for very high velocities. As this parameter is difficult to constrain, especially for large impacts, we consider here that it is a free parameter.

During the impact, a shock wave propagates from the impact site. Following the adiabatic pressure release, the peak pressure being almost independent of impactor size, a thermal anomaly remains below the impact site. The heat deposition is nearly uniform in a hemispherical (for $v_{i m p}<1 \mathrm{~km} / \mathrm{s}$ ) to spherical region next to the impact (the isobaric core), and strongly decays away from it (Croft, 1982; Squyres et al., 1988; Senshu et al., 2002) (see Fig. 1). For simplicity, we consider in our models that the shape of the isobaric core is spherical and that it does not depend on the impact velocity. Energy balance calculations and shock simulations suggest that, for impact velocities lower than $10 \mathrm{~km} . \mathrm{s}^{-1}$, the radius of the isobaric core $r_{i c}$ is comparable or slightly larger than that of the impactor $r_{i m p}$ (Pierazzo et al., 1997; Senshu et al., 2002; Kraus et al., 2011). Considering the extreme case in which all of the impact energy is perfectly transferred to the internal energy within the isobaric core and impactor itself gives an estimation of the maximum value for $r_{i c} / r_{i m p}=3^{1 / 3}$ (Senshu et al., 2002). Hence, after a large impact, a large amount of heat can be buried deep below the impact site at a depth $\sim 2 r_{i m p}$ and contribute to the early thermal 
evolution of the growing moon (Kraus et al., 2011).

As already explained in the introduction, we neglect here the velocity at infinity of the impactor $\left(v_{\infty}=0\right)$ as we want to determine the maximal size a moon can reach without significant melting. For simulations presented here, we do not consider any transplanetary impactor with $v_{i m p} \gg v_{e s c}$ (Squyres et al., 1988). The impactor velocity is only determined by the gravitational acceleration of the growing target: $v_{i m p}=v_{e s c}=\sqrt{2 g R}$ with $g$ the gravity at the surface of a moon with radius $R$. The impactor velocity is therefore proportional to the satellite size. For isobaric core volume $V_{i c}=3 V_{i m p}$, a balance between the kinetic energy delivered to the growing moon and the energy used to heat up the growing moon (isobaric core and the material surrounding it) without melting leads to (Monteux et al., 2007):

$$
\Delta T_{0}=\frac{4 \pi}{9} \frac{\gamma_{l i} \rho G{\overline{R_{t}}}^{2}}{h_{m} C_{p}}
$$

where $\rho$ is the mean density of the moon, $h_{m}$ represents the volume effectively heated normalized by the volume of the isobaric core and scales with the power $m$ (see values in Tab. 1). $\gamma_{l i}$ is the fraction of the impactor kinetic energy that is used to heat up the deep material of the impacted body. Hence, the postimpact temperature increase scales with the square of the moon radius at the time of impact (see Eq.1). Using parameter values from Tab. 1 and $\gamma_{l i}=30 \%$, for an impacted body with a radius ranging from $1000 \mathrm{~km}$ to $2500 \mathrm{~km}, v_{i m p}<3$ $\mathrm{km} / \mathrm{s}$ and $\Delta T_{0}$ ranges from $\sim 10 \mathrm{~K}$ to $100 \mathrm{~K}$. Obviously, if the velocity at infinity is non negligible, the delivered energy and hence temperature increase would be higher. However, as we want to determine the maximum radius that a growing satellite can reach without significant melting, we consider the most favorable case where the velocity at infinity is zero. 
Away from the isobaric core the peak pressure decays with the distance from the surface of the isobaric core (Pierazzo et al., 1997; Kraus et al., 2011) (see Fig. 1). This pressure decay can be faster for an ice/rock mixture than for terrestrial material because of the ice properties (Kraus et al., 2011). Just after the adiabatic pressure release, the thermal perturbation corresponds to an isothermal sphere of radius $r_{i c}$ and temperature $T_{0}+\Delta T_{0}$ that decays when $\bar{r}>r_{i c}$ as (see Fig. 1)

$$
T(r)=T_{0}+\Delta T_{0}\left(\frac{r_{i c}}{\bar{r}}\right)^{m}
$$

where $\bar{r}$ is the distance from the centre of the isobaric core, $T_{0}$ is the preimpact temperature and $m$ is the power characterizing the temperature decrease from the isobaric core (Pierazzo et al., 1997; Senshu et al., 2002). The postimpact temperature increase is a function of the pressure increase below the impact site. For small impact velocities (i.e. $<3 \mathrm{~km} . \mathrm{s}^{-1}$ ), the pressure $P$ may increase to peak values of $8 \mathrm{GPa}$ and the post-impact temperature increase scales with $P^{0.7-1}$ (Stewart and Ahrens, 2005). As the pressure typically decays from the isobaric core with $\sim\left(r_{i c} / r\right)^{4}$ (Kraus et al., 2011), the post impact temperature increase decays from the isobaric core following $\left(r_{i c} / r\right)^{m}$ with $m$ ranging from 2.8 to 4 . In this study we choose a medium value of $m=3.4$.

\subsection{Topographical effect}

An impact leads to the formation of a transient cavity of diameter $D_{s}$, reaching its final size $D_{f}$ after some modifications. The diameter of the transient crater $D_{s}$ can be related to the impactor diameter $d_{i m p}$ (in $\mathrm{km}$ ) through (Zahnle et al., 2003): 


$$
D_{s}=a_{0}\left(\frac{v_{i m p}^{2}}{v_{e s c}^{2}}\right)^{a_{1}}\left(\frac{\rho_{i m p}}{\rho}\right)^{a_{2}} R^{a_{3}} d_{i m p}^{a_{4}} \cos (\theta)^{a_{5}}
$$

211

212

where $v_{i m p}$ is the impactor velocity, $v_{e s c}$ is the escape velocity of the impacted moon, $\rho_{i m p}$ is the impactor density, $R$ is the radius of the moon (in $\mathrm{km}$ ) and $\theta$ is the impact angle. For simplicity, we assume $\rho_{i m p}=\rho$ and we set $\theta=45^{\circ}$ (the most likely angle of impact and the average value for a uniform bombardment (Shoemaker, 1962)). $a_{0}, a_{1}, a_{2}, a_{3}, a_{4}$ and $a_{5}$ are constant values listed in Table 2. These are derived from laboratory experiments as well as numerical modelling, and are consistent with planetary surface observations.

If the transient crater diameter is smaller than a critical value $D_{c}$, no later significant modifications occur and its final diameter is $D_{f}=D_{c}$. Among the parameters listed in Table $2, D_{c}$ is the one that exhibits the largest range of values as this parameter depends on the mechanical properties and gravity of each icy moon (McKinnon et al., 1991; Zahnle et al., 2003). $D_{c}$ typically ranges between 2-3 km for Ganymede and Callisto and up to $15 \mathrm{~km}$ for most of the medium-sized satellites (Schenk et al., 2004). Hence, $D_{c}$ is expected to vary during the growth of the icy moon. Here for simplicity we consider a single value, $D_{c}=15 \mathrm{~km}$ (see Table 2 ). In our models, the majority of the impacts leads to the formation of craters that are larger than $D_{c}$. Above $D_{c}$, the postimpact strength of the target material is insufficient to prevent collapse under gravity, crater modifications occur, resulting in a complex crater with a flat floor, a central peak or peak ring, and a terraced rim. Its final diameter thus becomes:

$$
D_{f}=D_{s}\left(\frac{D_{s}}{D_{c}}\right)^{b_{0}}
$$


We express the maximal depth at the centre of the crater $z_{f}$ as a function of the transient simple crater diameter (Pike, 1977; Schenk, 1991):

$$
z_{f}=\left\{\begin{array}{lll}
K_{1} D_{s}^{b_{1}} & \text { if } & D_{s}<D_{c} \\
K_{2} D_{s}^{b_{2}} & \text { if } & D_{s}>D_{c}
\end{array}\right.
$$

235 236 (Schenk, 1991):

$$
\delta_{0}=K_{3} D_{f}^{b_{3}}
$$

${ }_{237} b_{0}, b_{1}, b_{2}$ and $b_{3}$ are constant values listed in Tab. 2. The elevation variation $\xi_{i m p}$ the impact latitude. 

$269 z_{f}$.

\subsection{Ejected material and ejecta temperature}

The fraction of material from the impactor and from the impacted body escaping the growing moon decreases with decreasing impact velocities (Asphaug, 2010; Korycansky and Zahnle, 2011). For impact velocities considered in our models $\left(v_{i m p}=v_{e s c}<3 \mathrm{~km} \cdot \mathrm{s}^{-1}\right)$ and for $45^{\circ}$ impact angle, the accretion is supposed to be efficient and this fraction should remain small (less than $10 \%$ of the impactor's mass) (Asphaug, 2010; Korycansky and Zahnle, 2011). After a large impact, part of the material beneath the impact site is excavated and redeposited within the ejecta rim (see Fig. 1). We thus set $n$ from Eq.7 to a value typically ranging between 2 and 3 in order for the efficiency of mass accretion to be close to $100 \%$ during the whole accretion period and we consider that the whole impactor is deposited in the ejecta rim.

The temperature of this material depends on the pre-impact temperature, the temperature increase from the impact and the temperature of the impactor. The volume fraction of excavated material that is shock-heated increases with final crater size and this hot material is redeposited in the most external part of the ejecta rim (Maxwell, 1977; Barnhart and Nimmo, 2011). Hence, the thermal repartition within the ejecta rim should also depend on the interactions between the ejected material and the atmosphere during the excavation and the fallback processes (Kieffer and Simonds, 1980). For simplicity, we will consider in our models that the temperature of the ejecta rim is the average temperature below the impact site over a cylindrical volume with a diameter $D_{f}$ and a thickness 


\section{Multi-impact approach}

The accretion of an icy moon is the result of material deposited from a wide range of impactor sizes (i.e. from dusts to $100 \mathrm{~km}$ size objects). In the following sections we describe our model of accretion from multi-impacts.

\subsection{Impactor population}

For the mass distribution of the impactor, we consider a power law distribution with an exponent equal to $-2.5: d N_{c} / d m \propto m^{-2.5}$, consistent with N-body simulations (Kokubo and Ida, 2000). We use Monte Carlo sampling to generate the impactor population (Zahnle et al., 2001; Lognonné et al., 2009). By random drawing, we determine the impactor mass (or equivalently, radius) according to the above power law distribution. The time of impact is taken from a uniform probability distribution, while the latitude and longitude of the crater center are randomly drawn so that an isotropic impact flux is obtained. To limit the computation time, a lower size limit, $r_{m i n}$, is imposed on the impactor distribution (see Fig. 2). Below this lower limit, individual impact events are not simulated and a parameterized approach using successive deposit layers is used (see section 3.3 for further details). We assumed a lower limit, $r_{m i n}$, typically between 1 and $10 \mathrm{~km}$. We also prescribed an upper limit, $r_{\max }$, typically $100-$ $200 \mathrm{~km}$. Above these values, the validity of the scaling laws used here becomes questionable. Accretion from such large bodies would require more complex impact simulations, which is beyond the scope of the present paper. Nevertheless, $200 \mathrm{~km}$ is probably a reasonable upper limit since the growing moon is likely to perturb large objects that were migrating in from the outer disk possibly leading to their breakup. Hyperion, for instance, may be considered as an example of such large satellitesimals (Mosqueira and Estrada, 2003a,b; Estrada et al., 2009). The probability of impacts with objects exceeding $200 \mathrm{~km}$ is thus likely low, except maybe during the very late stage of accretion (e.g., Sekine 
and Genda, 2012).

For simplicity, the impactor population is assumed to be infinite (meaning that the number of impactors of a given size does not decrease as a function of time) and the accretion rates of large impactors $\tau_{a c c, l i}$ and layer deposit $\tau_{a c c, l a y}$ are assumed constant during one simulation. To measure the influence of large impactors $\left(r_{\min }<r<r_{\max }\right)$ relative to small impactors $\left(r<r_{\min }\right)$, we define the ratio:

$$
x_{m, l i}=m_{l i} / m_{a c c}
$$

where $m_{l i}$ is the mass accreted from large impactors and $m_{a c c}$ is the total mass accreted. We define the total accretion rate $\tau_{a c c}$ as

$$
\tau_{a c c}=\tau_{a c c, l i}+\tau_{a c c, l a y}
$$

where $\tau_{a c c, l i}$ is the accretion rate from large impacts and $\tau_{a c c, l a y}$ is the accretion rate from small impactors modelled as thin layer deposits (see section 3.3). We assume that the composition of the icy moon (and of the impactor) is a mixture of ice and rocks and that its density $\rho$ is uniform with depth.

\subsection{Multi-impact-induced topography}

To account for the pre-impact topography, we use the multi-cratering approach developed by Howard (2007). At the $i_{t h}$ impact, the new elevation variation $\Delta E_{i}(\eta, \xi)$ is

$$
\Delta E_{i}(\eta, \xi)=\left\{\begin{array}{ccc}
\Delta H(\eta, \xi)+\left(R_{i-1}(\eta, \xi)-\overline{R_{i-1}}\right)\left(1-\left(2 r / D_{f}\right)^{2}\right) & \text { when } & r<D_{f} / 2 \\
\Delta H(\eta, \xi)+\left(R_{i-1}(\eta, \xi)-\overline{R_{i-1}}\right) & \text { when } & r>D_{f} / 2
\end{array}\right.
$$


$\Delta E_{i}(\eta, \xi)$ depends on the local pre-impact topography variation $\left.\left(R_{i-1}(\eta, \xi)\right)-\overline{R_{i-1}}\right)$.

We consider here no late deformation of the topography before the impact (the degree of inheritance is 1 inside and outside the crater (Howard, 2007)). After the $i_{t h}$ impact, the local radius becomes $R_{i}(\eta, \xi)=R_{i-1}(\eta, \xi)+\Delta E_{i}(\eta, \xi)$ and the mean radius of the growing moon increases from $\overline{R_{i-1}}$ to $\overline{R_{i}}$.

The growth of the satellite requires that at least part of the impactor material remains on the growing satellite. Since we consider that the volume of the impactor is retained within the ejecta rim in our models, this growth requirement provides constraints on the scaling law describing the ejecta blanket distribution. For large $n$ values, the topography decreases rapidly from the crater rim and the volume of material accumulated in the ejecta rim decreases. On the contrary, for small $n$ values and for the same crater rim height, the topography decreases more linearly from the crater rim and the volume of material accumulated in the ejecta rim is large. The falloff in ejecta thickness is steep. Depending on the target properties, $n$ ranges between 2.5 and 3 (Housen et al., 1983; Moore et al., 2004). In Fig. 3, we monitor the average radius of the growing moon as a function of time for different values of $n$ and compare it with the theoretical mean radius resulting from the $100 \%$ accretion of $1.4 \times 10^{6}$ impactors ranging from 10 to $100 \mathrm{~km}$ radii. From this figure, we see that increasing $n$ decreases the mass accumulated and leads to a growth that is less than $100 \%$ accretive. For $n=3$, the accretion is not fully efficient and about $30 \%$ of the impacted mass remains on the impacted body while for $n=2.5,95 \%$ is accreted (see Fig. 3). For $n$ values smaller than 2.5, the growth is unrealistic since it is more than $100 \%$ accretive. We choose a value of 2.5 which maximize the fraction of accreted material. 


\subsection{Layer deposits from small impactors}

As explained previously, for numerical reasons, individual impact events for $r<r_{\min }$ are not simulated. We consider that the accreted mass from small impactors is averaged and uniformly added on the surface. For a prescribed accretion rate, $\tau_{a c c, l a y}=\tau_{a c c} \times\left(1-x_{m, l i}\right)$, the thickness $\delta_{\text {lay }}$ of the uniform layer deposit between two individual large impacts is then:

$$
\delta_{l a y}=\left(\frac{3 \tau_{a c c, l a y} \Delta t}{4 \pi \rho}+R_{i}^{3}\right)^{1 / 3}-R_{i}
$$

At any point at the surface, this additional layer is added uniformly. We assume that the temperature of this deposit layer is homogeneous over the entire thickness $\delta_{l a y}$. The layer temperature depends on the radius of the growing moon $\overline{R_{t}}$ and is calculated following an approach that is similar to the "classic" one from Schubert et al. (1981). In their 1D thermal evolution models, Schubert et al. (1981) considered that a fraction $h$ of the kinetic energy accumulated during accretion progressively heats up the near surface of the growing satellite (Kaula, 1979; Schubert et al., 1981; Lunine and Stevenson, 1987; Grasset and Sotin, 1996). Hence the corresponding temperature profile is:

$$
T\left(\overline{R_{t}}\right)=\frac{h G M\left(\overline{R_{t}}\right)}{C_{p} \overline{R_{t}}}\left(1+\frac{\overline{R_{t}} v_{\infty}^{2}}{2 G M\left(\overline{R_{t}}\right)}\right)+T_{e}
$$

Considering that $v_{\infty}^{2}=0$ (i.e. $v_{i m p}=v_{e s c}$ ), Eq.13 becomes

$$
T\left(\overline{R_{t}}\right)=\frac{\gamma_{l a y}}{2 C_{p}} v_{i m p}^{2}+T_{e}
$$

where $C_{p}$ is the heat capacity of the icy satellite material/mixture and $T_{e}$ is the temperature of the surrounding environment. The coefficient $\gamma_{l a y}$ represents the fraction of energy that is retained in the layer as heat. Note that the coefficients $\gamma_{l i}$ and $\gamma_{l a y}$ defined here differ from the coefficient $h$ used in 
Eq.13. $h$ implicitly includes the post-impact surface cooling, while $\gamma_{l i}$ and $\gamma_{l a y}$ only represent the fraction of kinetic energy converted as heat from the small impacts deposited as an uniform layer $\left(\gamma_{l a y}\right)$ or from large impacts $\left(\gamma_{l i}\right) \cdot \gamma_{l a y}$ is considered as a free parameter. It accounts for the effect of mechanical mixing in the shallow layers which has been described in Squyres et al. (1988) by a larger thermal diffusivity. Due to the heat removal by this "gardening" effect of numerous small impacts (Davies, 2009), it is reasonable to assume that $\gamma_{l a y} \leq \gamma_{l i}$

\subsection{Numerical method}

As the satellite grows, impactors bring material and thermal energy used to build-up and heat-up the moon. We monitor the thermal evolution of a growing icy satellite using the 3D-tool OEDIPUS (Choblet et al., 2007) to obtain a three-dimensional solution of the energy equation in a spherical shell. We use a finite-volume formulation and a mesh based on the "cubed sphere" transformation, the resulting grid consisting in six identical blocks. The computational grid in one block consists typically of $128 \times 64 \times 64$ discrete cells. Initially, the growing satellite in our models consists of a core surrounded by a shell with a thickness leading to a $R_{0}$ radius body. In the numerical domain, the overlaying shell (between $R_{0}$ and the final moon radius) is initially empty and gradually filled by impacted material during the accretion history. As the accretion time is relatively short compared to the onset time of solid-state convection (e.g., Robuchon et al., 2010), we consider only the diffusion of heat with no advective term. Melt transport and water/rock separation are not considered here and simulations are stopped when a few percent of material exceeding the melting point of water ice is reached. The accreted material is assumed to be an undifferentiated mixture of ice and rocks with a thermal diffusivity that does not depend on temperature, $\kappa=10^{-6} \mathrm{~m}^{2} \cdot \mathrm{s}^{-1}$ (Squyres et al., 1988; Barr et al., 
389

2010).

To maintain an accurate spatial resolution in our models during the entire accretion, we subdivide the accretion in successive stages between which the mesh grid is modified. Between two stages, the temperature field from the previous regime is interpolated on the mesh grid that we use in the next regime (see Fig. 4). The free accretionary parameters of our models are the ratio of material accreted from a large impacts $x_{m, l i}$ and the accretion rate $\tau_{a c c}$. The free energy conversion factors are $\gamma_{l a y}$ (layer heating) and $\gamma_{l i}$ (large impact heating). $\gamma_{l a y}$ and $\gamma_{l i}$ are independent parameters.

\subsection{Post-impact surface cooling}

After an impact, the efficient radiative heat transfer at the surface leads to a rapid cooling of the uppermost part of the heated zone (including the impact site and the surrounding ejecta blanket). As such a rapid post-impact cooling cannot be properly described in the framework of the relatively coarse grid mesh used by the 3D OEDIPUS tool, we have implemented a more precise description of heat transfer in this region. In the uppermost grid mesh of OEDIPUS, the conduction of heat for uniform heat conductivity is solved in the radial direction using refined sublayers with a Crank-Nicholson method (similarly to Tobie et al. (2003)). The number of sub-layers varies between 50 and 150, depending on the distance between the local surface radius $R_{i}(\eta, \xi)$ and the first underlying OEDIPUS grid mesh. A radiative heat flux boundary condition is imposed at the surface:

$$
F=\sigma\left(T\left(R_{i}\right)^{4}-T_{\mathrm{eq}}^{4}\right)
$$

with $\sigma$ the Stefan-Boltzman constant and $T_{\text {eq }}$ the expected equilibrium surface temperature. In the calculations presented below, $T_{\text {eq }}=100 \mathrm{~K}$. The tem- 
413

414

perature at the base of the refined column correspond to the temperature value provided in OEDIPUS. The conductive heat flux predicted in the refined column at the base of the first underlying OEDIPUS mesh interface is then imposed as heat flux boundary conditions at the top of the coarse grid domain.

\section{Numerical results}

4.1. Early and intermediate regimes: from $100 \mathrm{~km}$ to $1000 \mathrm{~km}$

We first consider the accretion of a $1000 \mathrm{~km}$ size ice-rock body from a 100 $\mathrm{km}$ satellite embryo. For simplicity, the initial temperature from $R=30 \mathrm{~km}$ to $R=R_{0}=100 \mathrm{~km}$ is set to a uniform value, here $T=T_{e}=100 \mathrm{~K}$. To maintain a good spatial resolution, we subdivide the accretion history of the icy satellite in two stages: an early stage where the moon is growing from $100 \mathrm{~km}$ to 500 $\mathrm{km}$, an intermediate stage where the moon is growing from $500 \mathrm{~km}$ to $1000 \mathrm{~km}$.

Fig. 4 illustrates the temperature evolution during these two accretionary regimes. In order to test the influence of the early and intermediate regimes on the late accretive stage, we consider two accretionary different scenarios for both the early and intermediate stages: a "cold accretion" where $\gamma_{l i}=\gamma_{l a y}=0.1$, $x_{m, l i}=10 \%$ (Fig. 4, left column) and a "hot accretion" where $\gamma_{l i}=\gamma_{l a y}=0.3$, $x_{m, l i}=33 \%$ (Fig. 4, middle column). The accretion parameters used for the "Early regime" simulation are $r_{\min }=4 \mathrm{~km}$ and $r_{\max }=10 \mathrm{~km}$, while for the "Intermediate regime", we used $r_{\min }=8 \mathrm{~km}$ and $r_{\max }=20 \mathrm{~km}$. At the end of the intermediate regime, $t_{a c c}=0.5 \mathrm{Myr}$ and the impactor velocities remain small $\left(<1 \mathrm{~km} . \mathrm{s}^{-1}\right)$ which corresponds to small temperature increases deep below the impact site $(<10 \mathrm{~K})$.

When the moons reach a radius of $1050 \mathrm{~km}$, the temperature barely exceeds 
$120 \mathrm{~K}$ in the cold accretive case, while it can reach values up to $250 \mathrm{~K}$ (near the melting point of water ice) for the hot accretive scenario. As we will show later in section 4.2 , although the obtained temperature fields are very different in these two cases, this has no major influence on the evolution of the temperature field in the outer part above $1000 \mathrm{~km}$. Fig. 4 (third column) also represents the 3D topography at the surface of the icy moon at the end of the two stages. As we increase the $r_{\min }$ and $r_{\max }$ values between the two simulations, the impact craters become larger and the contrast in topography (the difference between the $R(\eta, \xi)$ and the mean radius $\bar{R})$ also increases.

\subsection{Late accretive regime: $>1000 \mathrm{~km}$}

To simulate the evolution for $R>1000 \mathrm{~km}$ (late accretion regime), we use the thermal state reached at the end of the intermediate regime as the initial thermal state. In Fig. 4 we show results obtained for the same accretionary parameters in the late regime $\left(\gamma_{l i}=0.1, \gamma_{l a y}=0.3, x_{m, l i}=33 \%, r_{\min }=10 \mathrm{~km}\right.$ and $r_{\max }=100 \mathrm{~km}$ ) but for different initial temperature fields: "cold accretion" scenario (left column) and "hot accretion" scenario (middle column) obtained at the end of the corresponding intermediate regime. Fig. 4 illustrates that the temperature field obtained from the intermediate regime (hot or cold accretion scenario) only plays a minor role on the critical radius from which melting becomes significant during the late regime. Using the intermediate thermal state obtained form the cold accretion regime leads to $R_{\text {crit }}=1609 \mathrm{~km}$ while using the intermediate thermal state obtained form the hot accretion regime leads to $R_{\text {crit }}=1608 \mathrm{~km}$ (Fig. 4, last line). For this reason, in the following, the temperature field and topography from the "hot accretion scenario" are considered as initial conditions for all simulations of the accretion of bodies larger than $1000 \mathrm{~km}$. 
As explained previously, we assume that the impactor velocity is only deter-

mined by the gravitational acceleration, and we specifically test the influence of (1) accretion rate $\tau_{a c c},(2)$ mass fraction provided by large impactors $x_{m, l i}$ and (3) energy conversion factors (i.e. $\gamma_{l i}$ and $\gamma_{l a y}$ ) on the thermal state of the growing moon. We monitor the temperature field evolution as well as the volume fraction of satellite material that reaches the melting temperature of pure water ice (i.e. with $T>273 \mathrm{~K}$ ) as a function of satellite growth (see Fig. 4). As complex physical processes associated with melting and water-rock separation are beyond the scope of the present study, we interrupt the simulations when the volume fraction of the growing moon where $T>T_{\text {melt }}$ exceeds a threshold value fixed to $5 \%$ here. We define $R_{c r i t}$ as the satellite radius at which this threshold is reached. In this "late regime", the accretionary parameters can be different from the values used in the previous regimes which may lead to temperature "discontinuities" within the growing moon as emphasized in Fig. 4). As indicated above, such artefacts do not influence the value of $R_{\text {crit }}$. As illustrated in Fig. 4, the regions where melting occurs (the regions where the temperature scale is saturated in white) are mainly confined in the most external parts of the growing moon.

4.3. Influence of the accretion rate, $\tau_{a c c}$ and of the fraction of large impactors, $x_{m, l i}$

For this simulation, we assume that the conversion rate of impact energy is similar for small and large impactors: $\gamma_{l i}=\gamma_{l a y}=30 \%$ or $10 \%$. and we focus only on the late accretive regime. From our models, we can measure the influence of large impacts relative to layer deposition of small impactors by varying the value of $x_{m, l i}$. Fig. 5 shows the evolution of $R_{c r i t}$ as a function of $x_{m, l i}$ and for three different accretion rates. For a better comparison with other studies, 
509

we express the accretion rate, $\tau_{a c c}$, in terms of $M_{\text {Titan }} /$ Myr where $M_{\text {Titan }}$ is the mass of Titan $\left(=1.345 \times 10^{23} \mathrm{~kg}\right)$ and we consider values ranging between 0.015 $M_{\text {Titan }} /$ Myr $\left(=2 \times 10^{15} \mathrm{~kg} \cdot \mathrm{yr}^{-1}\right)$ and $1.5 M_{\text {Titan }} /$ Myr $\left(=2 \times 10^{17} \mathrm{~kg} \mathrm{yr}^{-1}\right)$. $\tau_{a c c} \leq 1.5 \mathrm{M}_{\text {Titan }} / \mathrm{Myr}$ corresponds to a relatively slow accretion, which is commonly assumed for the accretion of Callisto (Mosqueira and Estrada, 2003a; Barr and Canup, 2008).

Fig. 5 shows that, even for the least efficient conversion rate of impact energy $\left(\gamma_{l i}=\gamma_{l a y}=10 \%\right)$, the satellite cannot grow above $1500 \mathrm{~km}$ without significant melting, if the accretion is dominated by large impactors $\left(x_{m, l i} \sim 1\right)$. For $\gamma_{l i}=\gamma_{l a y}=30 \%$, the critical radius is even below $1200 \mathrm{~km}$. The critical radius can be increased only if a significant fraction of small impactors $(<10 \mathrm{~km})$ is considered. However, even if small impactors dominate, the critical radius does not exceed $1400 \mathrm{~km}$ if $\gamma_{l i}=\gamma_{l a y}=30 \%$. The critical radius can exceed 2000 $\mathrm{km}$ only if $\gamma_{l a y}=10 \%$ and if at least $50 \%$ of the accreted mass is brought by small impactors $\left(x_{m, l i}<0.5\right)$.

The accretion rate has some influence on the results only if the accretion is dominated by small impactors, as the rate at which new layers are added limits the cooling of the previously accreted layers. For simulations dominated by large impactors, as most of the energy is buried a few kilometers below the surface, the cooling is very inefficient and the progressive temperature increase only weakly depends on the accretion rate. Therefore, the size distribution of impactors is more crucial than the accretion rate in controlling the thermal evolution of growing satellites. However, as illustrated by the comparison between $\gamma_{l i}=$ $\gamma_{l a y}=10 \%$ and $\gamma_{l i}=\gamma_{l a y}=30 \%$ in Fig. 5, the energy conversion rate remains the most crucial parameters, and we explore in more details the sensitivity of 
our results to $\gamma_{l a y}$ and $\gamma_{l i}$ in the next subsection.

\subsection{Influence of the energy conversion parameters, $\gamma_{l a y}$ and $\gamma_{l i}$}

As shown in Fig. 6, for $x_{m, l i}=33 \%$ and $\tau_{a c c}=0.15 M_{\text {Titan }} / M y r, \gamma_{l a y}$ and $\gamma_{l i}$ must be smaller than 0.12 to allow the accretion of a body larger than 2000 $\mathrm{km}$ without significant melting. Conversion parameters as low as 0.1 correspond to the lowest value usually considered in previous studies (e.g., Squyres et al., 1988; Coradini et al., 1995). Such low values could be obtained for small impactors, but are probably a strong underestimation for large impactors. Fig. 6 also illustrates the relatively weak influence of the mean density on the thermal evolution of the growing moon. A decrease in the average density leads to a decay of the impact-induced temperature increase (see Eq.1). As a consequence, decreasing $\rho$ by $25 \%$ increases $R_{\text {crit }}$ by $\sim 15 \%$.

Fig. 7 shows the influence of increasing the energy conversion rate associated to large impactors, $\gamma_{l i}$ for a fixed value of $\gamma_{l a y}(=0.1)$ for small impactors and for three different values of $x_{m, l i}$. As expected, the critical radius strongly decreases when the conversion rate and the mass fraction associated to large impactors are increased. For $\gamma_{l i}=0.3$ (Fig. 8), the critical radius never exceeds $1600 \mathrm{~km}$. Fig. 9 represents the stability domain of a growing icy moon with $x_{m, l i}=33 \%$ and $\tau_{a c c}=0.15 M_{\text {Titan }} /$ Myr for different values of $\gamma_{l a y}$ and $\gamma_{l i}$. From Fig. 9, we see that, for $\gamma_{l i} \sim 0.3$ (O'Keefe and Ahrens, 1977; Squyres et al., 1988; Monteux et al., 2007) melting is more likely to occur as soon as the growing moon reaches a radius of $1200-1500 \mathrm{~km}$ which is in good agreement with Estrada and Mosqueira (2011). According to Fig. 9, it is difficult to envision a cold accretion as soon as $\gamma_{l a y}$ is larger than 0.3 even with small $\gamma_{l i}$. However, we may envision that the icy moon grows unmelted up to a radius of $1200 \mathrm{~km}$ even with $\gamma_{l i}>0.5$ only if $\gamma_{l a y}$ is smaller than 0.15 . 
571

\section{Conclusion}

We have developed a 3D numerical model that accounts for the influence of large impacts on the thermal evolution of growing icy satellites and have considered the least efficient scenarios and parameters to initiate melting. Our results show that the size distribution of impactors (i.e. ratio between large and small impactors) is a key factor in determining the temperature increase during the accretion stage. We show that the accretion rate as well as the thermal state of the satellite embryo only play a minor role, therefore the apparent degree of differentiation of a satellite's interior cannot be used to constrain the duration of its accretion.

Our simulations confirm that the most crucial parameter is the coefficient of impact energy conversion into heat $\left(\gamma_{l a y}\right.$ and $\left.\gamma_{l i}\right)$. Our results show that it is impossible to avoid significant melting during accretion, unless the fraction of impact energy retained as heat is very low, in the order of $10 \%$. Such an inefficient conversion rate is difficult to explain and does not seem realistic with respect to available estimates from impact experiments (e.g., Ahrens and Okeefe, 1985). Much lower initial temperature of the impactors as well as more efficient subsurface cooling associated with impact gardening (not modelled explicitly here but included in the $\gamma_{l a y}$ conversion efficiency) may reduce the effective conversion rates (Anderson, 1989). Lower environment temperature $(<100 \mathrm{~K})$ may also increase the cooling rate of the shallow layers. Therefore, the absence of extensive melting during accretion may reflect a very cold ambient subnebula rather than a long accretionary timescale.

Several additional heat sources such as radiogenic heating, tidal/despinning heating or heating associated with high-velocity impact, have not been consid- 
ered in the heat budget in our model. Including these would require an even less efficient energy conversion and storage to avoid melting and subsequent differentiation. We also made the conservative assumption that the impacts are $100 \%$ accretive. If some fraction of impact is not fully accretive, more impacts are needed to accrete the same mass resulting in more impact energy. Hence, the temperature increase would be higher and melting even more likely. Therefore, the maximal radii of the accreted satellite reached without significant melting in our simulations can be considered as upper limits.

Based on our simulations, when more than $10 \%$ of the accreted mass is brought by impactors larger than $1 \mathrm{~km}$, it seems unlikely that a satellite larger than $2000 \mathrm{~km}$ may accrete without significant melting unless the environment is extremely cold and the conversion rate of impact energy unrealistically low $(<10-15 \%)$. If the accretion is dominated by km-size impactors, impactinduced melting may occur for radii as small as 1100-1500 km. Above this critical radius, separation between liquid water and rock should initiate, thus leading to the accumulation of dense rock blocks above the undifferentiated core consisting of a mixture of rock and ice (e.g., Kirk and Stevenson, 1987). The dense layer of accumulated rock is gravitationally unstable, and in such conditions it is difficult to avoid subsequent full separation of rock and ice phases. Depending on the size of the core and thickness of the rocky layer, the differentiation may be catastrophic (Kirk and Stevenson, 1987) or more gradual (Nagel et al., 2004). Recently, O'Rourke and Stevenson (2013) showed that although rock-ice separation may be delayed by double-diffusive convection in the ice-rock interior, ice melting due to progressive radiogenic heating and subsequent differentiation cannot be prevented. Further modelling efforts are needed to better understand the processes controlling rock-ice segregation and how the internal 
structure inherited from the accretion process has evolved to the present-day state.

A series of arguments now questions the apparent partially differentiated state of Callisto and Titan, suggested by their elevated moment of inertia as estimated using the Radau-Darwin Approximation (e.g., Anderson et al., 2001; Iess et al., 2010; Gao and Stevenson, 2013). On Titan, the existence of a nonnegligible degree-three in the gravity field as well as significant topography suggest that non-hydrostatic effects may significantly affect the estimation of the Moment-of-Inertia factor (Iess et al., 2010; Gao and Stevenson, 2013; Baland et al., in revision) and that the $\mathrm{MoI}$ factor may be significantly smaller than the value estimated from the Radau-Darwin Approximation. On Callisto, similar non hydrostatic contributions originating in the lithosphere may also affect the estimation of its moment of inertia (McKinnon, 1997; Gao and Stevenson, 2013).

On these two moons, the hydrostatic dynamical flattening is relatively small as they orbit relatively far from their planet, and therefore the non-hydrostatic contributions need to be correctly estimated in order to accurately infer the moment of inertia and the density profile of their interior. On Callisto, future measurements performed by the ESA JUICE mission that will be launched in 2022 (Grasset et al., 2013) will provide constraints on the non-hydrostatic contribution by measuring independently the different quadrupole coefficients, as well as by estimating the degree three and four coefficients of the gravity field. On Titan, future measurements during Cassini flybys will also permit a better determination of the degree-four (Iess et al., 2012), which will provide pertinent tests on the topography compensation process in the outer ice shell (Hemingway et al., 2013; Lefevre et al., 2014), and consequently on the non-hydrostatic corrections required to infer more precisely the moment of inertia. 


\section{Acknowledgements}

The authors also thank the anonymous reviewers for constructive comments. J. Monteux is funded by Agence Nationale de la Recherche (Accretis decision no. ANR-10-PDOC-001-01). The research leading to these results has received funding from the European Research Council under the European Community's Seventh Framework Programme (FP7/2007- 2013 Grant Agreement no. 259285).

\section{References}

Ahrens, T. J., and J. D. Okeefe (1985), Shock vaporization and the accretion of the icy satellites of Jupiter and Saturn, in NATO ASIC Proc. 156: Ices in the Solar System, edited by J. Klinger, D. Benest, A. Dollfus, and R. Smoluchowski, pp. 631-654.

Anderson, D. (1989), Theory of the Earth, Chap.: The Terrestrial Planets, Boston: Blackwell Scientific Publications.

Anderson, J. D., R. A. Jacobson, T. P. McElrath, W. B. Moore, G. Schubert, and P. C. Thomas (2001), Shape, Mean Radius, Gravity Field, and Interior Structure of Callisto, Icarus, 153, 157-161, doi:10.1006/icar.2001.6664.

Asphaug, E. (2010), Similar-sized collisions and the diversity of planets, Chemie der Erde / Geochemistry, 70, 199-219, doi:10.1016/j.chemer.2010.01.004.

Baland, R., G. Tobie, A. Lefevre, and T. Van Hoolst (in revision), Titan's internal structure inferred from its gravity field, shape, and rotation state, Icarus.

Barnhart, C. J., and F. Nimmo (2011), Role of impact excavation in distributing clays over Noachian surfaces, Journal of Geophysical Research (Planets), 116, E01,009, doi:10.1029/2010JE003629. 
Barr, A. C., and R. M. Canup (2008), Constraints on gas giant satellite formation from the interior states of partially differentiated satellites, Icarus, 198, 163-177, doi:10.1016/j.icarus.2008.07.004.

Barr, A. C., R. I. Citron, and R. M. Canup (2010), Origin of a partially differentiated Titan, Icarus, 209, 858-862, doi:10.1016/j.icarus.2010.05.028.

Canup, R. M., and W. R. Ward (2002), Formation of the Galilean Satellites: Conditions of Accretion, aj, 124, 3404-3423, doi:10.1086/344684.

Canup, R. M., and W. R. Ward (2006), A common mass scaling for satellite systems of gaseous planets, Nature, 441, 834-839, doi:10.1038/nature04860.

Castillo-Rogez, J. C., and J. I. Lunine (2010), Evolution of Titan's rocky core constrained by Cassini observations, Geophys. Res. Lett., 37, L20205, doi: 10.1029/2010GL044398.

Choblet, G., O. Cadek, F. Couturier, and C. Dumoulin (2007), (EDIPUS: a new tool to study the dynamics of planetary interiors, Geophysical Journal International, 170, 9-30, doi:10.1111/j.1365-246X.2007.03419.x.

Coradini, A., C. Federico, O. Forni, and G. Magni (1995), Origin and thermal evolution of icy satellites, Surveys in Geophysics, 16, 533-591, doi: $10.1007 / \mathrm{BF} 00665684$.

Croft, S. K. (1982), A first-order estimate of shock heating and vaporization in oceanic impacts, vol. 190, 143-152 pp., Geological Implications of Impacts of Large Asteroids and Comets on Earth, edited by T.L. Silver and P.H. Schultz,Spec. Pap. Geol. Soc. Am.

Davies, G. (2009), Thermal evolution of the mantle, Treatise of Geophysics, vol. 9, 197-216 pp., Schubert, G. editor in Chief, Elsevier. 
Dwyer, C., F. Nimmo, M. Ogihara, and S. Ida (2013), The influence of imperfect accretion and radial mixing on ice:rock ratios in the galilean satellites, Icarus, 225 (1), 390 - 402, doi:http://dx.doi.org/10.1016/j.icarus.2013.03.025.

Estrada, P. R., and I. Mosqueira (2011), Titan's Accretion and Long Term Thermal History, in Lunar and Planetary Institute Science Conference Abstracts, Lunar and Planetary Institute Science Conference Abstracts, vol. 42, p. 1679.

Estrada, P. R., I. Mosqueira, J. J. Lissauer, G. D'Angelo, and D. P. Cruikshank (2009), Formation of Jupiter and Conditions for Accretion of the Galilean Satellites, pp. 27-+, University of Arizona Press.

Gao, P., and D. J. Stevenson (2013), Nonhydrostatic effects and the determination of icy satellites' moment of inertia, Icarus, 226, 1185-1191, doi: 10.1016/j.icarus.2013.07.034.

Grasset, O., and C. Sotin (1996), The Cooling Rate of a Liquid Shell in Titan's Interior, Icarus, 123, 101-112, doi:10.1006/icar.1996.0144.

Grasset, O., et al. (2013), JUpiter ICy moons Explorer (JUICE): An ESA mission to orbit Ganymede and to characterise the Jupiter system, Planet. Space Sci., 78, 1-21, doi:10.1016/j.pss.2012.12.002.

Hemingway, D., F. Nimmo, H. Zebker, and L. Iess (2013), A rigid and weathered ice shell on Titan, Nature, 500, 550-552, doi:10.1038/nature12400.

Housen, K. R., R. M. Schmidt, and K. A. Holsapple (1983), Crater ejecta scaling laws - Fundamental forms based on dimensional analysis, J. Geophys. Res., 88, 2485-2499, doi:10.1029/JB088iB03p02485.

Howard, A. D. (2007), Simulating the development of Martian highland landscapes through the interaction of impact cratering, fluvial ero- 
sion, and variable hydrologic forcing, Geomorphology, 91, 332-363, doi: 10.1016/j.geomorph.2007.04.017.

Iess, L., N. J. Rappaport, R. A. Jacobson, P. Racioppa, D. J. Stevenson, P. Tortora, J. W. Armstrong, and S. W. Asmar (2010), Gravity Field, Shape, and Moment of Inertia of Titan, Science, 327, 1367-, doi:10.1126/science.1182583.

Iess, L., et al. (2012), The Tides of Titan, Science, 337, 457-, doi: 10.1126/science.1219631.

Kaula, W. M. (1979), Thermal evolution of earth and moon growing by planetesimal impacts, J. Geophys. Res., 84, 999-1008.

Kieffer, S. W., and C. H. Simonds (1980), The role of volatiles and lithology in the impact cratering process, Reviews of Geophysics and Space Physics, 18, 143-181, doi:10.1029/RG018i001p00143.

Kirk, R. L., and D. J. Stevenson (1987), Thermal evolution of a differentiated Ganymede and implications for surface features, Icarus, 69, 91-134, doi: 10.1016/0019-1035(87)90009-1.

Kivelson, M. G., J. Warnecke, L. Bennett, S. Joy, K. K. Khurana, J. A. Linker, C. T. Russell, R. J. Walker, and C. Polanskey (1998), Ganymede's magnetosphere: Magnetometer overview, J. Geophys. Res., 103, 19,963-19,972, doi:10.1029/98JE00227.

Kokubo, E., and S. Ida (2000), Formation of Protoplanets from Planetesimals in the Solar Nebula, Icarus, 143, 15-27, doi:10.1006/icar.1999.6237.

Korycansky, D. G., and K. J. Zahnle (2011), Titan impacts and escape, Icarus, 211, 707-721, doi:10.1016/j.icarus.2010.09.013. 
Kossacki, K. J., and J. Leliwa-Kopystyński (1993), Medium-sized icy satellites: thermal and structural evolution during accretion, Planet. Space Sci., 41, 729-741, doi:10.1016/0032-0633(93)90115-I.

Kraus, R., L. Senft, and S. Stewart (2011), Impacts onto h2o ice: Scaling laws for melting, vaporization, excavation, and final crater size, Icarus.

Lefevre, A., G. Tobie, G. Choblet, and O. Cadek (2014), Structure and dynamics of Titan's outer icy shell constrained from Cassini data, Icarus, p. in press.

Lognonné, P., M. Le Feuvre, C. L. Johnson, and R. C. Weber (2009), Moon meteoritic seismic hum: Steady state prediction, J. Geophys. Res. (Planets), 114, E12003, doi:10.1029/2008JE003294.

Lunine, J. I., and D. J. Stevenson (1987), Clathrate and ammonia hydrates at high pressure - Application to the origin of methane on Titan, Icarus, 70, 61-77, doi:10.1016/0019-1035(87)90075-3.

Maxwell, D. E. (1977), Simple Z model for cratering, ejection, and the overturned flap., in Impact and Explosion Cratering: Planetary and Terrestrial Implications, edited by D. J. Roddy, R. O. Pepin, and R. B. Merrill, pp. $1003-1008$.

McKinnon, W. B. (1997), NOTE: Mystery of Callisto: Is It Undifferentiated?, Icarus, 130, 540-543, doi:10.1006/icar.1997.5826.

McKinnon, W. B., C. R. Chapman, and K. R. Housen (1991), Cratering of the Uranian satellites, pp. 629-692, University of Arizona Press.

Monteux, J., N. Coltice, F. Dubuffet, and Y. Ricard (2007), Thermo-mechanical adjustment after impacts during planetary growth, Geophys. Res. Lett., 34, $24,201-24,205$. 
Moore, J. M., P. M. Schenk, L. S. Bruesch, E. Asphaug, and W. B. McKinnon (2004), Large impact features on middle-sized icy satellites, Icarus, 171, 421443, doi:10.1016/j.icarus.2004.05.009.

Mosqueira, I., and P. R. Estrada (2003a), Formation of the regular satellites of giant planets in an extended gaseous nebula I: subnebula model and accretion of satellites, Icarus, 163, 198-231, doi:10.1016/S0019-1035(03)00076-9.

Mosqueira, I., and P. R. Estrada (2003b), Formation of the regular satellites of giant planets in an extended gaseous nebula II: satellite migration and survival, Icarus, 163, 232-255, doi:10.1016/S0019-1035(03)00077-0.

Mueller, S., and W. B. McKinnon (1988), Three-layered models of Ganymede and Callisto - Compositions, structures, and aspects of evolution, Icarus, 76 , 437-464, doi:10.1016/0019-1035(88)90014-0.

Nagel, K., D. Breuer, and T. Spohn (2004), A model for the interior structure, evolution, and differentiation of Callisto, Icarus, 169, 402-412, doi: 10.1016/j.icarus.2003.12.019.

O'Keefe, J. D., and T. J. Ahrens (1977), Impact-induced energy partitioning, melting, and vaporization on terrestrial planets, in Lun. Planet. Sci. Conf., vol. 8, edited by R. B. Merril, pp. 3357-3374.

O'Rourke, P., and D. J. Stevenson (2013), Stability of ice/rock mixtures with application to a partially differentiated Titan, Icarus, p. in press, doi: 10.1016/j.icarus.2013.07.034.

Pappalardo, R. T., G. C. Collins, J. W. Head, III, P. Helfenstein, T. B. McCord, J. M. Moore, L. M. Prockter, P. M. Schenk, and J. R. Spencer (2004), Geology of Ganymede, pp. 363-396, Jupiter. The Planet, Satellites and Mag- 
netosphere, edited by Bagenal, F. and Dowling, T. E. and McKinnon, W. B., Cambridge Planetary Science.

Pierazzo, E., A. M. Vickery, and H. J. Melosh (1997), A Reevaluation of Impact Melt Production, Icarus, 127, 408-423.

Pike, R. J. (1977), Apparent depth/apparent diameter relation for lunar craters, in Lunar and Planetary Science Conference Proceedings, Lunar and Planetary Science Conference Proceedings, vol. 8, edited by R. B. Merril, pp. 3427-3436.

Robuchon, G., G. Choblet, G. Tobie, O. Cadek, C. Sotin, and O. Grasset (2010), Coupling of thermal evolution and despinning of early Iapetus, Icarus, 20\%, 959-971, doi:10.1016/j.icarus.2009.12.002.

Safronov, V. S. (1978), The heating of the earth during its formation, Icarus, 33, 3-12, doi:10.1016/0019-1035(78)90019-2.

Schenk, P. M. (1991), Ganymede and Callisto - Complex crater formation and planetary crusts, J. Geophys. Res., 96, 15,635, doi:10.1029/91JE00932.

Schenk, P. M., C. R. Chapman, K. Zahnle, and J. M. Moore (2004), Ages and interiors: the cratering record of the Galilean satellites, pp. 427-456, Jupiter. The Planet, Satellites and Magnetosphere, edited by Bagenal, F. and Dowling, T. E. and McKinnon, W. B., Cambridge Planetary Science.

Schubert, G., D. J. Stevenson, and K. Ellsworth (1981), Internal structures of the Galilean satellites, Icarus, 47, 46-59, doi:10.1016/0019-1035(81)90090-7.

Sekine, Y., and H. Genda (2012), Giant impacts in the Saturnian system: A possible origin of diversity in the inner mid-sized satellites, Plan. Space Sci., 63, 133-138, doi:10.1016/j.pss.2011.05.015.

Senshu, H., K. Kuramoto, and T. Matsui (2002), Thermal evolution of a growing Mars, J. Geophys. Res., 10\%, 1-13. 
Shoemaker, E. M. (1962), Interpretation of lunar craters, pp. 283-359, Academic Press, San Diego.

Sohl, F., M. Choukroun, J. Kargel, J. Kimura, R. Pappalardo, S. Vance, and M. Zolotov (2010), Subsurface Water Oceans on Icy Satellites: Chemical Composition and Exchange Processes, Space Sci. Rev., 153, 485-510, doi: $10.1007 / \mathrm{s} 11214-010-9646-\mathrm{y}$.

Squyres, S. W., R. T. Reynolds, A. L. Summers, and F. Shung (1988), Accretional heating of the satellites of Saturn and Uranus, J. Geophys. Res., 93, 8779-8794, doi:10.1029/JB093iB08p08779.

Stewart, S. T., and T. J. Ahrens (2005), Shock properties of $\mathrm{H}_{2} \mathrm{O}$ ice, Journal of Geophysical Research (Planets), 110, E03005, doi:10.1029/2004JE002305.

Tobie, G., G. Choblet, and C. Sotin (2003), Tidally heated convection: Constraints on Europa's ice shell thickness, Journal of Geophysical Research (Planets), 108, 5124, doi:10.1029/2003JE002099.

Ward, W. R., and R. M. Canup (2010), Circumplanetary Disk Formation, Astr. Journ., 140, 1168-1193, doi:10.1088/0004-6256/140/5/1168.

Zahnle, K., L. Dones, and H. F. Levison (1998), Cratering Rates on the Galilean Satellites, Icarus, 136, 202-222, doi:10.1006/icar.1998.6015.

Zahnle, K., P. Schenk, S. Sobieszczyk, L. Dones, and H. F. Levison (2001), Differential Cratering of Synchronously Rotating Satellites by Ecliptic Comets, Icarus, 153, 111-129, doi:10.1006/icar.2001.6668.

Zahnle, K., P. Schenk, H. Levison, and L. Dones (2003), Cratering rates in the outer Solar System, Icarus, 163, 263-289, doi:10.1016/S0019-1035(03)000484. 
Table 1: Typical parameter values for numerical models

\begin{tabular}{lcc}
\hline Moon radius & $R$ & $100-2000 \mathrm{~km}$ \\
Impactor radius & $r_{i m p}$ & $4-100 \mathrm{~km}$ \\
Isobaric core radius & $r_{i c}$ & $1500-2000 \mathrm{~kg} \mathrm{~m}^{-3}$ \\
$\begin{array}{l}\text { Average moon density } \\
\text { Mean heat capacity }\end{array}$ & $\rho$ & $1200 \mathrm{~J} \mathrm{~K}^{-1} \mathrm{~kg}^{-1}$ \\
$\begin{array}{l}\text { Environment temperature } \\
\text { Mean heat diffusivity }\end{array}$ & $T_{e}$ & $100 \mathrm{~K}^{-1}$ \\
$\begin{array}{l}\text { Large impact energy fraction } \\
\text { retained }\end{array}$ & $\kappa$ & $10^{-6} \mathrm{~m}^{2} \mathrm{~s}^{-1}$ \\
$\begin{array}{l}\text { Temperature power decrease } \\
\text { from the isobaric core }\end{array}$ & $\gamma_{l i}$ & $0.1-0.6$ \\
$\begin{array}{l}\text { Volume effectively heated } \\
\text { by impact }\end{array}$ & $m$ & 3.4 \\
$\begin{array}{l}\text { Layer deposit energy fraction } \\
\text { retained }\end{array}$ & $h_{m}$ & 5.8 \\
Gravitational constant & $\gamma_{l a y} \leq \gamma_{l i}$ & $\mathrm{G}$ \\
& & $0.1-0.3$ \\
\hline
\end{tabular}




\begin{tabular}{|c|c|c|}
\hline Parameter & Value & References \\
\hline$a_{0}$ & 1.1 & (Zahnle et al., 1998, 2003) \\
\hline$a_{1}$ & 0.217 & " \\
\hline$a_{2}$ & 0.333 & $"$ \\
\hline$a_{3}$ & 0.217 & $"$ \\
\hline$a_{4}$ & 0.783 & $"$ \\
\hline$a_{5}$ & 0.44 & $"$ \\
\hline$D_{c}$ & $15 \mathrm{~km}$ & (McKinnon et al., 1991) \\
\hline$b_{0}$ & 0.13 & " \\
\hline$K_{1}$ & 0.15 & (McKinnon et al., 1991; Zahnle et al., 2003) \\
\hline$b_{1}$ & 0.88 & " \\
\hline$K_{2}$ & 0.75 & " \\
\hline$b_{2}$ & 0.3 & $"$ \\
\hline$K_{3}$ & 0.017 & (Schenk, 1991) \\
\hline$b_{3}$ & 0.976 & $($ Schenk, 1991) \\
\hline$p$ & $2-3$ & (Howard, 2007) \\
\hline$n$ & $2-3.5$ & " \\
\hline
\end{tabular}

Table 2: Crater geometrical parameters used in our models. 
Topographical effects
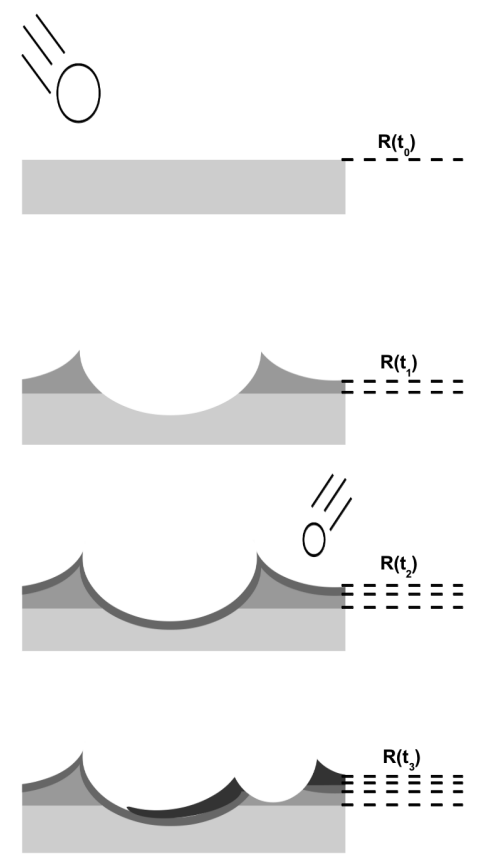

Thermal effects
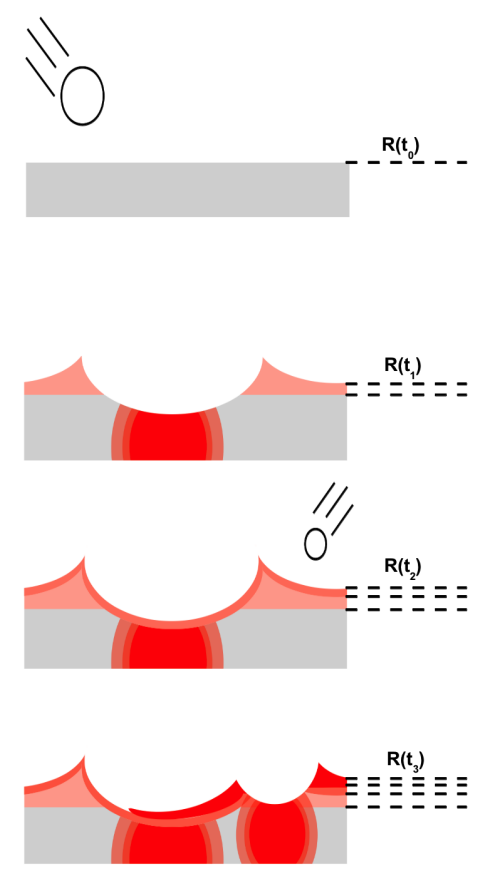

Figure 1: Schematic illustration of the topographical (left) and thermal (right) evolutions after large impacts. When the first large impact occurs (first line), a crater with diameter $D_{f}$, depth $z_{f}$ and rim height $\delta_{0}$ is formed (second line, left). Before the next large impact, the layer deposition occurs (third line, left). When a second impact occurs close enough to the first one (fourth line), the pre-existing topography is modified according to Eq.11. When a large impact occurs (first and second line, right), heat is buried deep below the impact site following Eq.1 while the ejecta rim temperature is the average temperature below the impact site over a volume that is $D_{f}$ large and $z_{f}$ thick. The temperature of the layer deposited before the next large impact (third line, right) obeys Eq.13. 


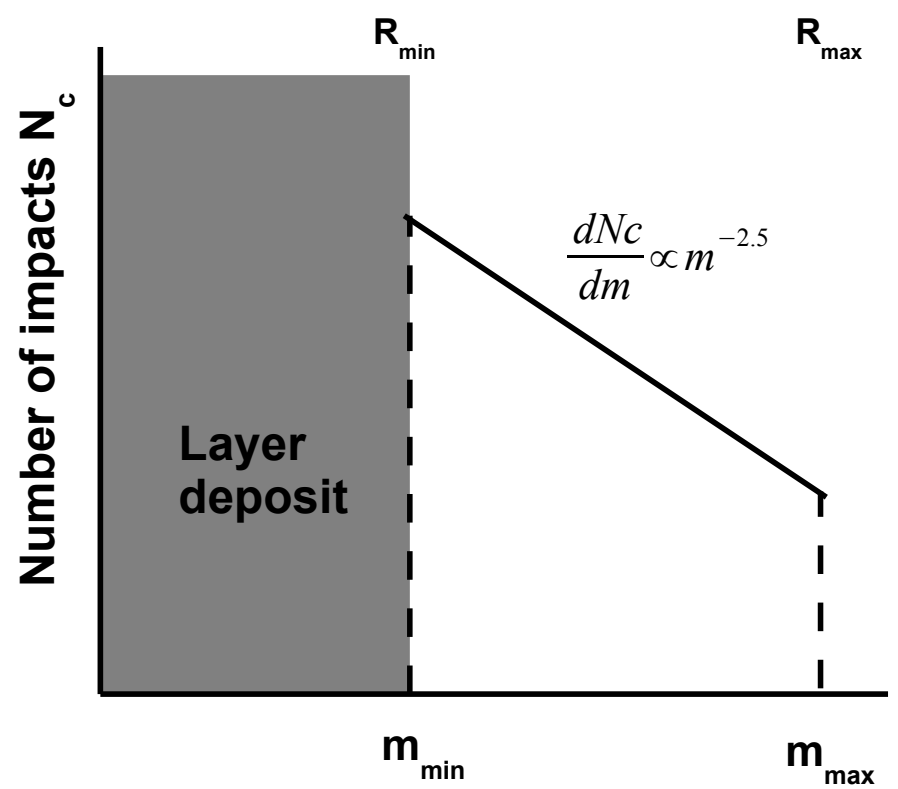

Impactor mass $\mathbf{m}$

Figure 2: Schematic representation of the cumulated number of impacts as a function of the impactor mass. All the material with a mass smaller than $m_{\min }$ (i.e. with $r<r_{\min }$ ) is deposited as a thin global layer over the moon surface. The impactors with a mass ranging from $m_{\min }$ and $m_{\max }$ are considered here as successive impact events (selected randomly) and their effects (impact cratering and heating) are treated individually. 


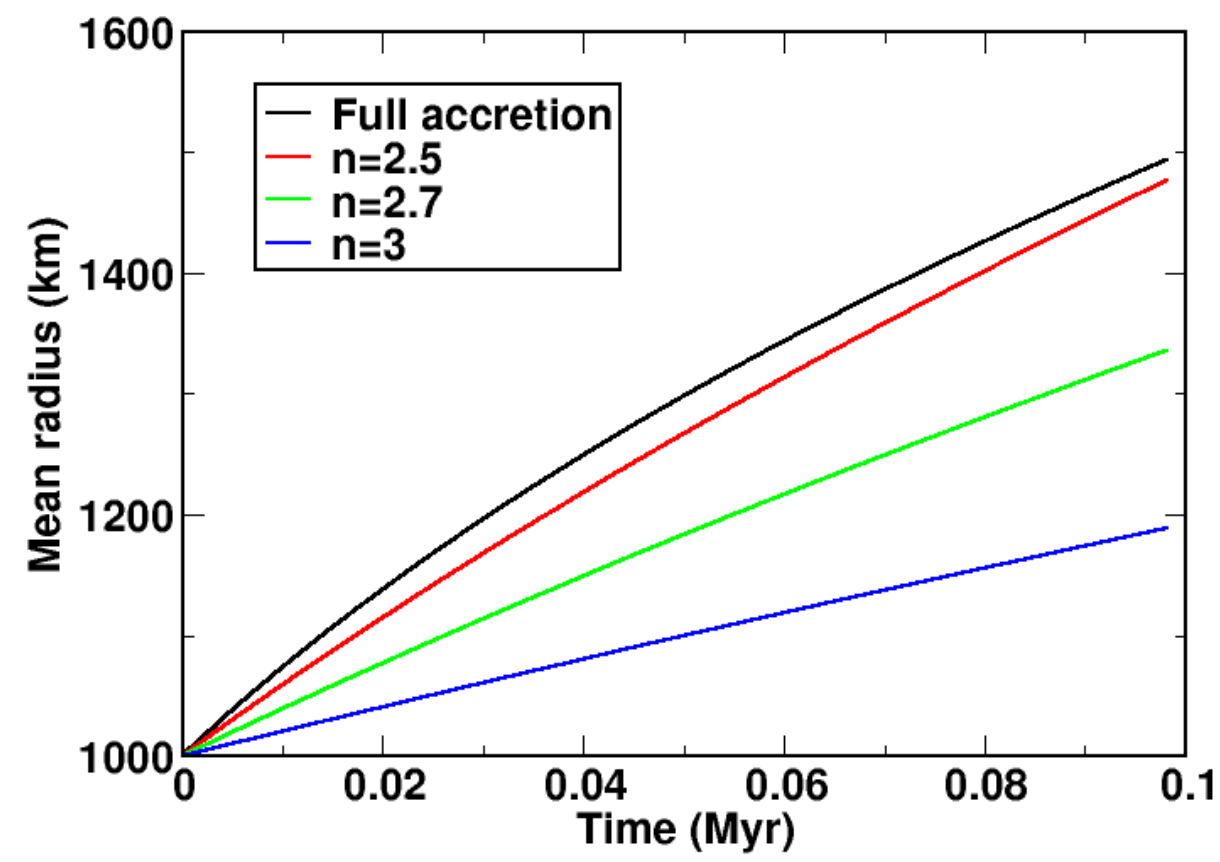

Figure 3: Time evolution of the average radius of the growing icy moon after the accretion of $1.4 \times 10^{6}$ impactors ranging from 10 to $100 \mathrm{~km}$ radii with $n=2.5$ (red solid line), $n=2.7$ (green solid line) and $n=3$ (blue solid line). For comparison, we also represent the time evolution of the average radius consisting in the $100 \%$ accretive accumulation of the impactor bodies (black solid line). 
"Cold accretion"

"Hot accretion"
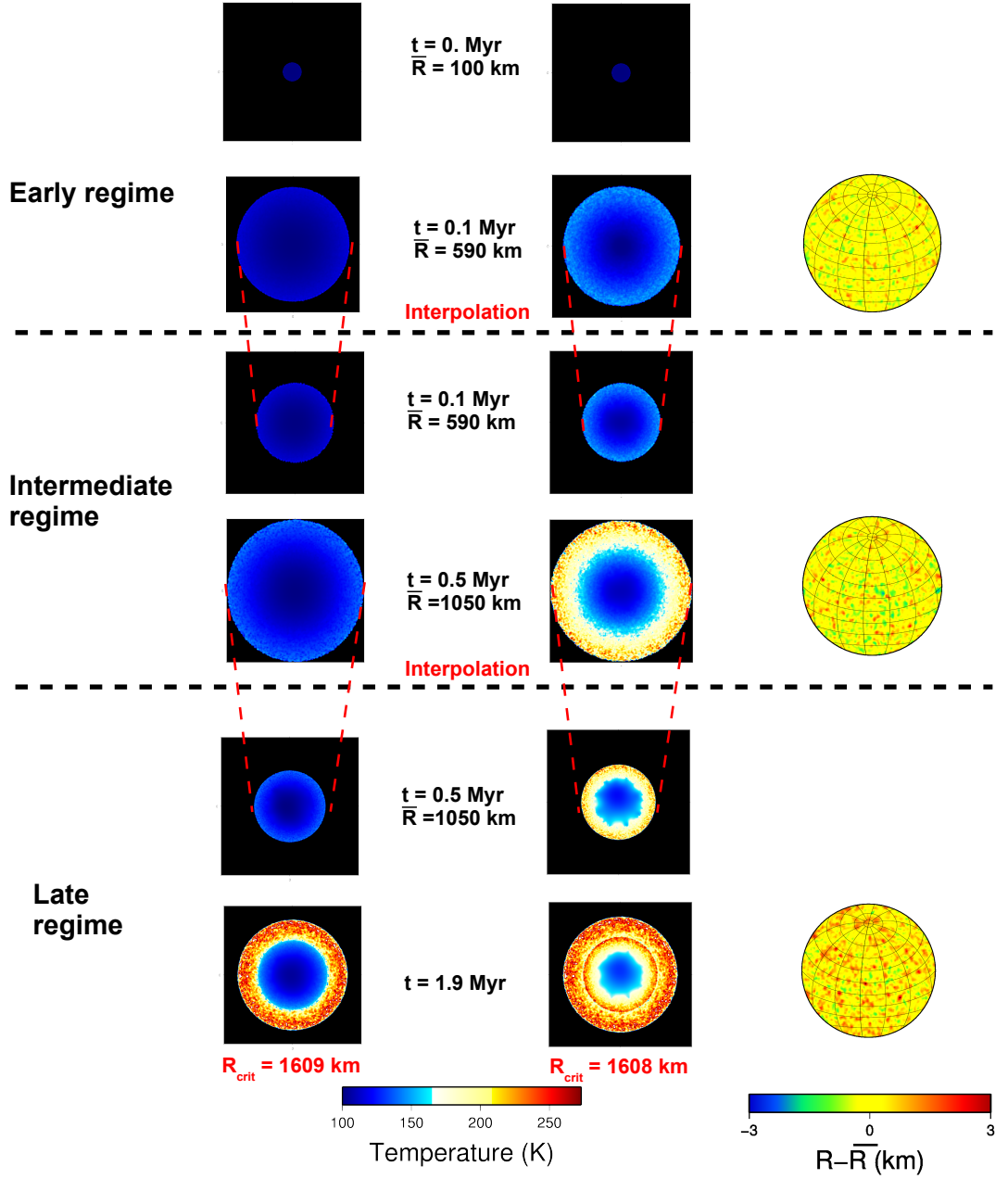

Figure 4: Equatorial cross sections of the temperature field (left and middle columns) and 3D topographical representations (right) of the growing icy moon as a function of time (from top to bottom). The left column represents the "cold accretion" evolution where, up to the end of the intermediate regime, $\gamma_{l i}=\gamma_{l a y}=0.1, x_{m, l i}=10 \%$ while the middle column represents the "hot accretion" evolution where $\gamma_{l i}=\gamma_{l a y}=0.3, x_{m, l i}=33 \%$ (Fig. 4, middle column). Temperature colour scale is saturated in white for temperature at the melting point $(>273$ $\mathrm{K})$. Between each regime (early, intermediate, late), the temperature field is interpolated to a larger mesh grid. In the "Late regime", $\gamma_{l i}=0.1, \gamma_{l a y}=0.3, x_{m, l i}=33 \%, r_{\min }=10 \mathrm{~km}$ and $r_{\max }=100 \mathrm{~km}$ for both the left and middle columns. 


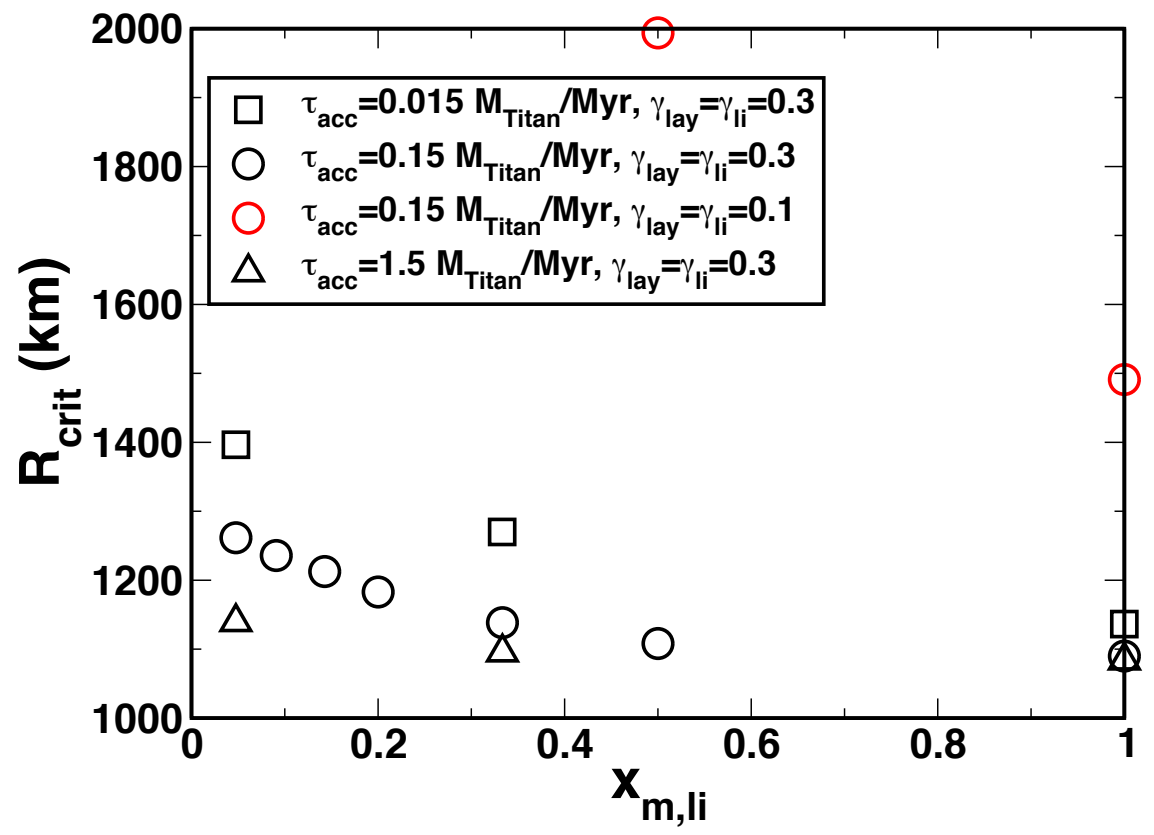

Figure 5: Critical radius $R_{\text {crit }}$ (above which more than $5 \%$ of the volume of the icy moon has a temperature larger than the melting temperature) as a function of the fraction of material accreted from large impacts $x_{m, l i}$ for different accretion rates ranging from $0.015 \mathrm{M}_{\text {Titan }} / \mathrm{Myr}$ to $1.55 M_{\text {Titan }} / M y r$. Black symbols represent $R_{\text {crit }}$ for $\gamma_{l a y}=\gamma_{l i}=0.3$ while red circles represent $R_{\text {crit }}$ for $\gamma_{l a y}=\gamma_{l i}=0.1$. 


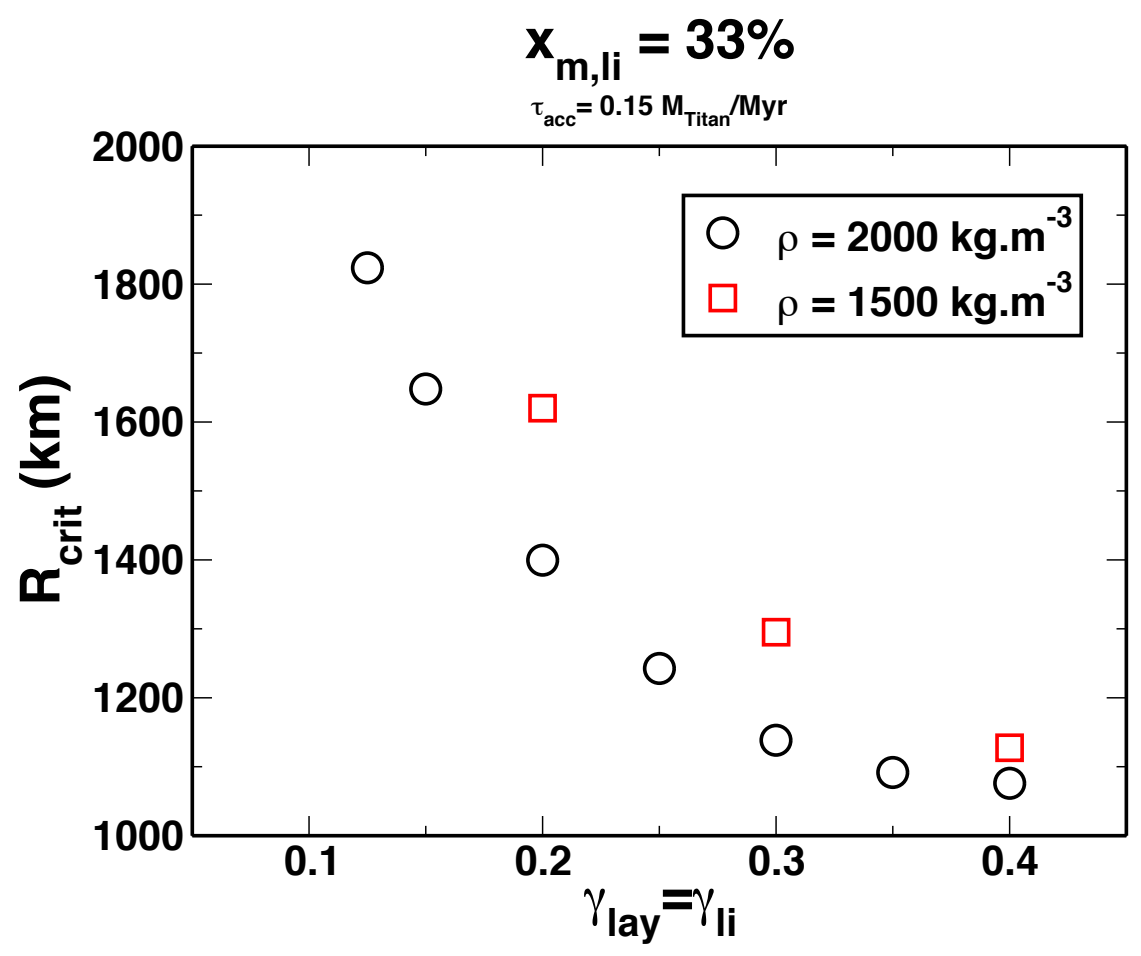

Figure 6: Critical radius $R_{\text {crit }}$ (above which more than $5 \%$ of the volume of the icy moon has a temperature larger than the melting temperature) as a function of the energy conversion coefficients $\left(\gamma_{l a y}\right.$ and $\left.\gamma_{l i}\right)$ for two density values $\left(\rho=1500 \mathrm{~kg} \cdot \mathrm{m}^{-3}\right.$ and $\left.\rho=2000 \mathrm{~kg} \cdot \mathrm{m}^{-3}\right)$. In these models, the energy conversion coefficients are set to be equal $\gamma_{l a y}=\gamma_{l i}$, the accretion rate is set to $0.15 M_{\text {Titan }} / M y r$ and the mass fraction of material accreted from large impacts is $x_{m, l i}=33 \%$. 


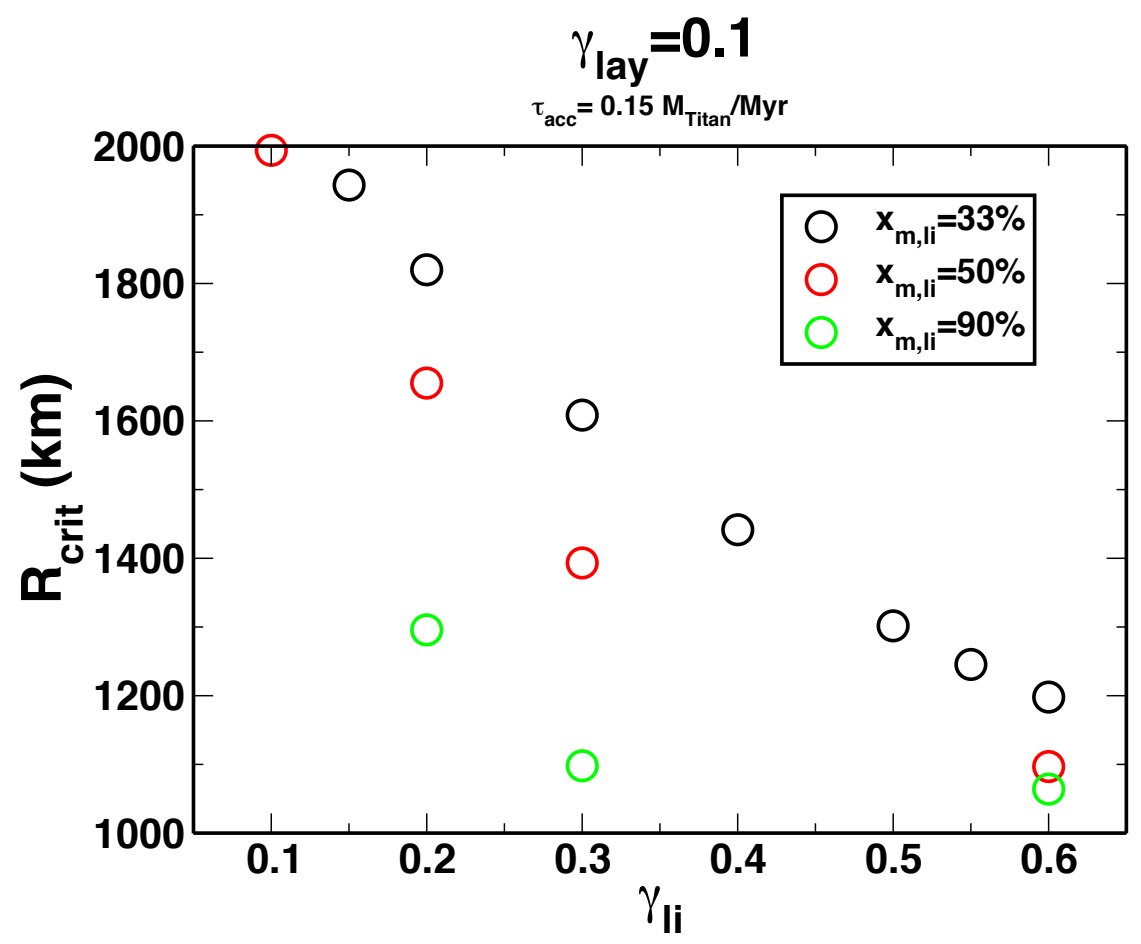

Figure 7: Critical radius $R_{\text {crit }}$ (above which more than $5 \%$ of the volume of the icy moon has a temperature larger than the melting temperature) as a function of the energy conversion coefficient $\gamma_{l i}$, for three values of $x_{m, l i}(33,50$ and $90 \%)$. In these simulations, the energy conversion coefficient $\gamma_{l a y}$ is set to $\gamma_{l a y}=0.1$ and the accretion rate is set to $0.15 \mathrm{M}_{\text {Titan }} / \mathrm{Myr}$. 


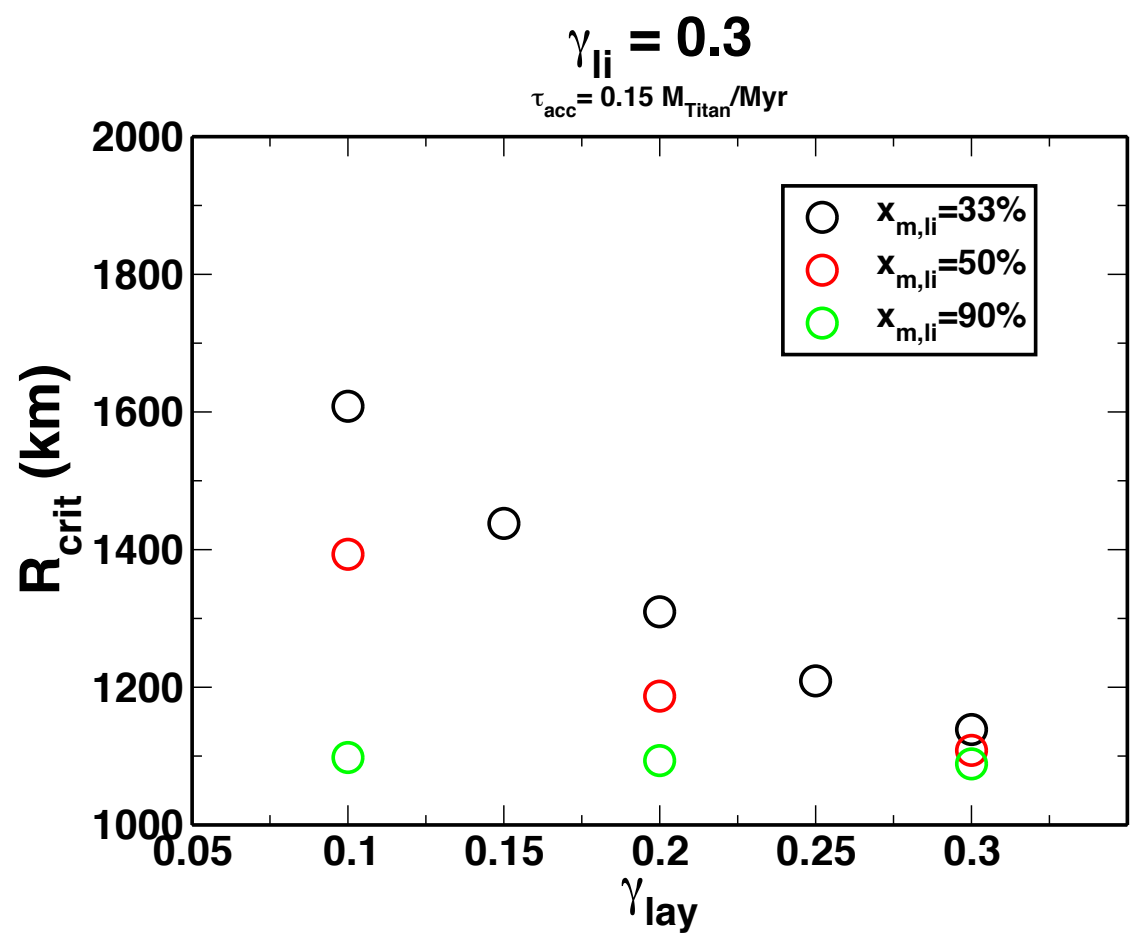

Figure 8: Critical radius $R_{\text {crit }}$ (above which more than $5 \%$ of the volume of the icy moon has a temperature larger than the melting temperature) as a function of the energy conversion coefficient $\gamma_{l a y}$ for three values of $x_{m, l i}(33,50$ and $90 \%)$. In these simulations, the energy conversion coefficient $\gamma_{l i}$ is set to $\gamma_{l i}=0.3$. We only represent the results with $\gamma_{l a y} \leq \gamma_{l i}$. The accretion rate is set to $0.15 M_{\text {Titan }} / M y r$. 


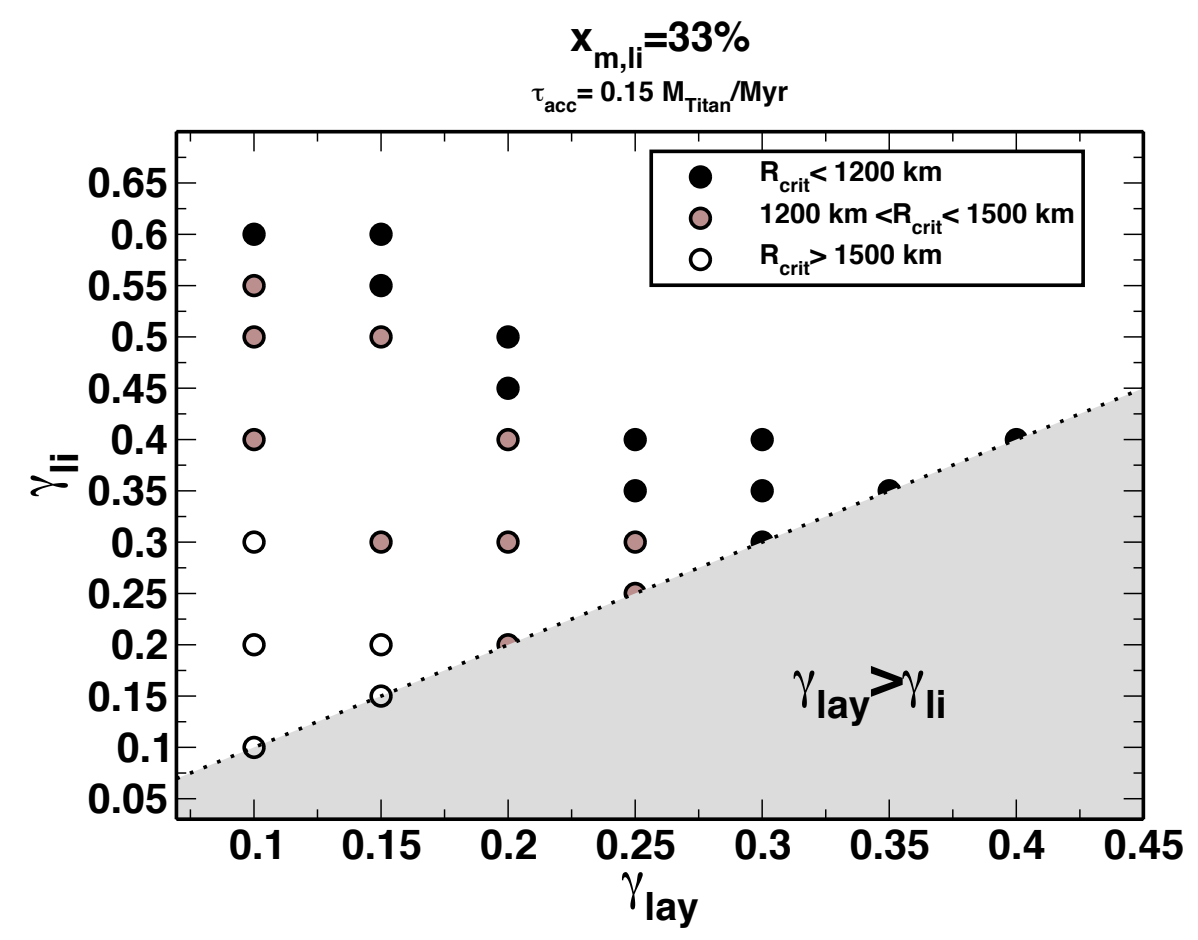

Figure 9: Melting behaviour of a growing icy moon as a function of the energy conversion coefficients $\gamma_{l a y}$ and $\gamma_{l i}$. For black-filled symbols, $R_{\text {crit }}<1200 \mathrm{~km}$. For brown-filled symbols, $1200<R_{\text {crit }}<1500 \mathrm{~km}$. For white-filled symbols, $R_{\text {crit }}>1500 \mathrm{~km}$. In these simulations, the accretion rate is set to $0.15 M_{\text {Titan }} / M y r$ and the mass fraction of material accreted from large impacts is $x_{m, l i}=33 \%$. In the grey domain, $\gamma_{l a y}>\gamma_{l i}$ and the corresponding cases are not considered here. 